\title{
Efficacy of a natural mineral complex in North American adults with osteoarthritis of the knee: a randomized double-blind placebo-controlled
} study

\author{
Malkanthi Evans \\ Dale Wilson \\ Najla Guthrie \\ KGK Synergize Inc., London, \\ ON, Canada
}

Correspondence: Malkanthi Evans KGK Synergize Inc., 255 Queens Ave, Suite 1440, London, ON N6A 5R8, Canada

Tel +I 5194389374 ext 239

Fax+I 51943883|4

Email mevans@kgksynergize.com
This article was published in the following Dove Press journal:

Open Access Rheumatology: Research and Reviews

3 October 2014

Number of times this article has been viewed

Purpose: This study evaluated the efficacy of a hydrothermal mineral complex (HMC) supplement in participants with knee osteoarthritis.

Patients and methods: This was a double-blind, placebo-controlled, 12-week crossover study with 150 participants receiving either placebo or HMC for 4 weeks, with a 4-week washout period. The primary endpoint was WOMAC ${ }^{\mathrm{TM}}$ pain, and secondary endpoints were WOMAC ${ }^{\mathrm{TM}}$ physical function and stiffness, the 36-Item Short Form Health Survey (SF-36), high-sensitivity C-reactive protein, tumor necrosis factor $\alpha$, interleukin 6 , and safety.

Results: There were no significant differences in WOMAC ${ }^{\mathrm{TM}}$ pain, stiffness, or physical function scores between groups. Within groups, subjects on both HMC and placebo reported improvements $(P<0.001)$ in all WOMAC ${ }^{\mathrm{TM}}$ domains. HMC performed significantly better in total SF-36 scores $(P=0.05)$ and physical function $(P=0.02)$, and had improved total physical activity $(P=0.06)$ and social functioning $(P=0.09)$ scores compared with placebo. Within groups, physical function ( $P=0.01)$, limitations due to mental health/emotional well-being $(P=0.02)$, bodily pain $(P=0.001)$, and total physical $(P=0.003)$ and mental health scores $(P=0.02)$ improved in participants on HMC, whereas improvements in bodily pain $(P=0.001)$, general health $(P=0.01)$, and total physical activity $(P=0.04)$ were reported in placebo. Subjects on HMC with body mass index $(\mathrm{BMI})<25 \mathrm{~kg} / \mathrm{m}^{2}$ showed a trend toward decreased pain scores $(P=0.10)$, while pain increased in those administered placebo. Minimal clinically important improvement (MCII) in WOMACTM pain scores increased from $28 \%$ of HMC-administered participants at week 2 to $41 \%$ at week 4 , and decreased to $37 \%$ after 2 weeks of washout. In comparison, $41 \%$ of placebo-administered subjects achieved MCII by week 2 and week 4. A 10.4\% greater increase in tumor necrosis factor $\alpha$ levels was seen in participants receiving placebo than those receiving $\operatorname{HMC}(P=0.07)$. There were no differences between groups in adverse events.

Conclusion: HMC significantly improved physical function and total physical activity, improving the quality of life of participants. HMC was most effective in normal-weight subjects Increased dosage may be required for North American subjects with BMI $>25 \mathrm{~kg} / \mathrm{m}^{2}$.

Keywords: knee osteoarthritis, WOMACTM, SF-36, hydrothermal mineral complex

\section{Introduction}

Osteoarthritis (OA) affects approximately $34 \%$ of people over 65 years of age and compromises the quality of life of more than 21 million North Americans. ${ }^{1,2} \mathrm{OA}$ accounts for $25 \%$ of visits to primary care physicians and costs the North American economy approximately US\$60 billion annually., ${ }^{2,3}$ Due to an increasing elderly population 
in North America, OA is becoming a major medical and financial concern. While there is a strong association between age and OA, risk factors such as obesity and joint injury can also lead to premature development of OA in younger adults. ${ }^{2,4}$ Activities resulting in excessive repetitive joint loading, such as repetitive lifting of heavy objects, continuously repeated movements, high-intensity and high-impact and repetitive athletics, as well as power and team sports, such as soccer, basketball, boxing, and weightlifting, have also been linked to early-onset OA. ${ }^{2,4}$

Although the risk factors of OA have been well documented, the pathophysiology of the joint resulting in the clinical signs and symptoms of OA is still not understood. ${ }^{2}$ OA can affect any synovial joint, but it occurs commonly in hand, knee, and hip joints. ${ }^{2}$ It is characterized by the degeneration of a synovial joint resulting from the progressive loss in articular cartilage, abnormal remodeling of the subarticular bone, and the formation of bone cysts and osteophytes. ${ }^{2,4}$ Primary OA, referred to as such when the cause of joint degeneration is not known, is rarely diagnosed in patients below 40 years of age. ${ }^{2}$ Secondary OA, on the other hand, is the development of disease after trauma or injury to the affected joint, or the result of a preexisting hereditary, inflammatory, developmental, metabolic, or neurologic disorder. ${ }^{2,5}$ OA of the knee is often associated with pain in and around the joint, stiffness, crepitation, and limited joint motion. ${ }^{6}$ The progression of OA is slow, and treatment of OA includes exercise, heat/cold therapy, joint protection, weight loss, physiotherapy/occupational therapy, and medication. ${ }^{7}$ Relieving or improving joint pain and stiffness and overall physical function are current recommendations for managing OA and are an important focus and goal of therapy. ${ }^{8,9}$

The most common medications for OA include acetaminophen and nonsteroidal anti-inflammatory drugs (NSAIDs) such as cyclo-oxygenase II (COX-2) inhibitors. These medications are effective in reducing pain associated with $\mathrm{OA}$ but do not prevent disease progression. Additionally, there are many serious potential side-effects associated with NSAIDs, including upper gastrointestinal tract problems, hypertension, congestive heart failure, and renal insufficiency. ${ }^{10-12}$ Furthermore, COX-2 inhibitors may increase the risk of platelet aggregation and myocardial infarction when compared to NSAIDs. ${ }^{13}$ The high incidence of NSAID-related adverse events, as well as a growing aging population, has increased the need and urgency for more effective and safer alternative treatments.

Complementary and alternative medicines (CAMs) are being increasingly sought after by consumers to alleviate
OA-associated pain. One in every two Australians, one in every three in the United States, and one in every four people in the United Kingdom are reported to be seeking CAMs for the treatment of their OA. ${ }^{14}$ Studies on the use of CAMs have reported that $\mathrm{OA}$ patients in the US are among the highest users of CAMs. ${ }^{15,16}$

In a recent Cochrane review on herbal therapy for treating OA, the authors identified only five out of approximately 2,500 citations that fully met their inclusion criteria. ${ }^{11}$ The problems revolved around methodology, inadequate sample size and length of study, large placebo effects, and poor quality of reporting. The plethora of poor quality studies in the literature, as well as large placebo effects seen in OA research, makes it difficult to draw reasonable conclusions regarding the efficacy of short- and long-term use of many pharmaceuticals and natural health products.

Hydrothermal mineral complex (HMC) (SierraSil Joint Formula $14^{\circledR}$; SierraSil Health Inc., Vancouver, BC, Canada) is a natural mineral complex containing a wide array of minerals in a silicate clay-like structure. HMC has been found to exert an anti-inflammatory effect by inhibiting the proinflammatory cytokine interleukin (IL)-1 $\beta$ and reducing nitric oxide production in vitro, and is thought to reduce cartilage damage. ${ }^{17} \mathrm{HMC}$ has previously been shown to improve joint health and function in a South Asian population of subjects with mild to moderate knee OA. ${ }^{18}$

In an in vitro model of OA, HMC limited cartilage destruction and activated chondrocytes to promote a healthy cartilage matrix. ${ }^{17}$ Furthermore, HMC was shown alone and in combination with cat's claw extract to significantly improve WOMAC ${ }^{\text {TM }}$ scores in subjects with mild to moderate knee OA. ${ }^{18}$

The objective of this study was to assess the clinical efficacy of HMC using the WOMAC ${ }^{\text {TM }}$ OA index, the 36-Item Short Form Health Survey (SF-36), and the minimal clinically important improvement (MCII) for WOMACTM pain on the symptoms of knee OA in a North American population of subjects.

\section{Material and methods Study design and participants}

This was a 12-week, randomized, double-blind, placebocontrolled, crossover study on the efficacy of HMC ( $2 \mathrm{~g} /$ day) in adults with OA of the knee with a 4-week washout period. The study was conducted in accordance with the International Conference on Harmonization Good Clinical Practice (ICH-GCP) guidelines and the ethical principles of the Declaration of Helsinki (2000). The study was reviewed 
by the Natural Health Products Directorate (NHPD) of Health Canada and a change from weight-dependent dosage to standard dosage was requested by NHPD. With this change, approval was received on August 25, 2011. The study protocol and materials were reviewed and approved by Institutional Review Board Services (Aurora, ON, Canada) on September 15, 2011. The results of this study are presented in line with the CONSORT (Consolidated Standards of Reporting Trials) guidelines. ${ }^{19}$

Voluntary, written informed consent was obtained from all subjects prior to participation in any study-related procedures. Subjects were considered eligible if they met the following criteria: were between 18 and 65 years of age; exhibited primary or secondary, unilateral or bilateral OA of the knee as defined by the American College of Rheumatology (ACR) Clinical Criteria for Classification (knee pain with at least three of the following: age greater than 50 years, stiffness less than 30 minutes, crepitus, bone tenderness, bone enlargement, and no palpable warmth); self-reported difficulty performing at least one of the following activities because of knee pain - lifting and carrying groceries, walking one-quarter of a mile, getting in and out of a chair, going up and down stairs, mobility, self-care activities, ability to walk unassisted; and if using other therapies for OA, such as exercise, heat/cold therapy, joint protection, and physiotherapy/occupational therapy, agree to continue these therapies and agree not to start any new therapies.

Subjects were considered ineligible if they had a diagnosis of rheumatoid arthritis, fibromyalgia, spinal disorders or any other musculoskeletal disease, had been recommended for knee surgery, had a planned surgery, used corticosteroids (intra-articular, oral, or parenteral) or other injectable prescription medication within 2 months prior to randomization and during the trial, required the use of prescription drugs to control pain, required the use of oral or topical prescription or over the counter medications or natural health products for pain relief, had clinically significant abnormal laboratory results at screening, or had an allergy or sensitivity to the test product ingredients.

Eligible subjects were randomized to HMC or placebo via a randomization schedule in a 1:1 ratio in blocks of two, with 75 subjects randomized to each of the two treatments. The investigational product was labeled according to the requirements of ICH-GCP guidelines and applicable local regulatory guidelines. Each product was labeled with similar labels, differing only by randomization number. The investigational product was dispensed at baseline and weeks 2, 8, and 10. Unused product was returned during subsequent visits. Participants were instructed to take one capsule of investigational product for 4 weeks, three times a day (morning, noon, and evening) with water only, and to drink 6-8 glasses of water daily.

In order to evaluate primary and secondary objectives, study assessments were conducted at baseline, week 2 , and week 4 of each treatment period. The screening visit included a review of medical history and concomitant therapies, and measurement of safety parameters. Inclusion/exclusion criteria were reviewed, and fasting blood samples were obtained for safety analysis. The target knee to be followed for the duration of the study was selected, and subjects completed the WOMAC ${ }^{\text {TM }}$ OA Index.

Randomized subjects returned to the clinic for Test Period 1 in a fasted condition for baseline assessments. The first dose of study product was taken the day following the baseline visit. Subjects returned to the clinic at week 2 and week 4 after baseline in a fasted condition for safety and efficacy assessments. Subjects returned to the clinic at week 6 for their first posttreatment visit when rescue medication and diary were returned and a new supply of rescue medication and diary dispensed. Subjects returned to the clinic at week 8 for Test Period 2. Subjects received the opposite treatment regimen to what they received during Test Period 1, but all procedures for the second test period were similar to the first test period. Clinic visits were at weeks 8, 10, and 12 . Subjects returned to the clinic at week 14 for their second period posttreatment visit.

HMC capsules contained $667 \mathrm{mg}$ of sierra silicate clay mineral with microcrystalline cellulose and magnesium stearate. The placebo contained brown rice flour, natural caramel powder, and colorants (FD\&C Yellow \#5 Lake, FD\&C Yellow \#40 Lake, FD\&C Blue \#1 Lake). Both products were encapsulated in vegetarian capsules of hydroxypropyl methylcellulose and purified water, and were the same size, shape, and color. In the event of a participant's OA symptoms becoming intolerable, rescue medication (acetaminophen $500 \mathrm{mg}$, up to $4 \mathrm{~g} /$ day) was provided. Participants were instructed to use rescue medication at their discretion and according to label guidelines of 1-2 tablets every 4 hours as required and not to exceed 8 tablets daily. Participants were instructed to record the amount of investigational product and rescue medication taken each day in their subject diaries.

Participants returned unused test products at each visit, and their compliance was calculated as the number of capsules that were taken divided by the number of capsules 
expected to have been taken, multiplied by 100. In the event of a discrepancy between the information recorded in the subject diary and the amount of study product or rescue medication returned, calculations were based on the product returned unless an explanation for loss of product was provided. Participants with compliance of less than $80 \%$ or greater than $120 \%$ at any visit were counseled. A compliance of less than $70 \%$ or greater than $130 \%$ was considered noncompliant, and any participant demonstrating noncompliance for two consecutive visits was withdrawn from the study.

\section{Outcome measures}

The primary endpoint was the efficacy of HMC on the symptoms of OA as assessed by the change in the WOMAC visual analog scale pain scores (range: 0-500). Secondary outcome measures were changes in the WOMAC ${ }^{\text {TM }}$ stiffness (range: 0-200) and physical function (range: 0-1,700) scores, the total SF-36 and individual domain scale scores, MCII, and serum concentration of the inflammatory markers high-sensitivity C-reactive protein (hsCRP), IL-6, and tumor necrosis factor (TNF)- $\alpha$. TNF- $\alpha$ and IL-6 were quantified using a commercial ELISA (enzyme-linked immunosorbent assay) kit according to the manufacturer's instructions (BD Biosciences, Mississauga, ON, Canada), and hsCRP was quantified by LifeLabs Medical Laboratory Services (London, ON, Canada). The WOMACTM index was completed every 2 weeks during the study, and the SF-36 questionnaire was completed at baseline and week 4 of each test period. Serum inflammatory markers were measured at baseline and every 2 weeks during each treatment period.

\section{Safety assessments}

Safety measurements were taken on all subjects at screening and after 2, 4, 8, and 12 weeks of supplementation. These included hematology and clinical chemistry, weight, heart rate, and blood pressure. A urine pregnancy test was performed on all eligible females at screening, and a physical examination was performed on all subjects at baseline (week 0) and included weight, heart rate, and blood pressure measurements. Adverse events were recorded in subject diaries and reviewed by the medical director at each study visit.

\section{Statistical methods}

A sample size calculation was performed to detect a betweentreatment difference of $30 \mathrm{~mm}$ for WOMAC ${ }^{\mathrm{TM}}$ pain based on $\alpha=0.05$ (two-sided) and $90 \%$ power for a standard deviation of 75 and allowing for a $20 \%$ withdrawal rate. It was determined that a sample size of 150 subjects was required, with 75 subjects equally assigned to treatment and placebo groups. Probability values $\leq 0.05$ were considered statistically significant. Statistical analysis was performed for participants who completed both treatment periods of the study. Participant demographics were compared between groups using a $t$-test or chi-square test as appropriate. Efficacy data were evaluated according to a crossover design. Between-treatment comparisons were made using repeated measures analysis of variance. Each parameter was tested first for potential period and carryover effects. Within-groups comparisons were made by paired $t$-test.

Subgroup analysis was planned and performed for WOMAC ${ }^{\text {TM }}$ subscales categorizing subjects by body mass index (BMI), sex, and age. Between-groups analysis for these comparisons were made using Wilcoxon (Mann-Whitney) nonparametric test, and within-group comparisons were made by paired $t$-test.

The number of participants meeting MCII for WOMAC ${ }^{\text {TM }}$ pain (improvement of $\geq 40.8 \%)^{20}$ was compared between treatments using Fisher's exact test.

Safety parameters, including biometrics, vital signs, hematology and clinical chemistry parameters, were compared by analysis of variance. Adverse events were compared using a chi-square test. All statistical analyses were performed using R software.

\section{Results \\ Participants}

A total of 150 subjects, 52 males and 98 females, were randomized in this study (Figure 1), with an average age of 45 years and BMI of $30 \mathrm{~kg} / \mathrm{m}^{2}$ (Table 1 ). Over $92 \%$ of participants were Caucasian and of Western-European origin. Seventy-five percent of the enrolled participants had primary OA, while $25 \%$ had secondary OA. A greater number of participants over 45 years of age had primary OA $(88 \%)$, while $12 \%$ had secondary OA. A similar trend was seen in participants below 45 years of age, where $59 \%$ had primary OA and $41 \%$ had secondary OA.

The mean age of participants sequencing from placebo to $\mathrm{HMC}$ was $45 \pm 13$ years (Table 1). Twenty-three percent were normal weight (BMI <25), 36\% were overweight (BMI 25.0-29.9), and 41\% were obese (BMI >30). The participants sequencing from HMC to placebo had a mean age of $45 \pm 12$ years, and $25 \%$ were normal weight (BMI $<25$ ), $31 \%$ overweight (BMI 25.0-29.9), and 44\% obese (BMI >30). 


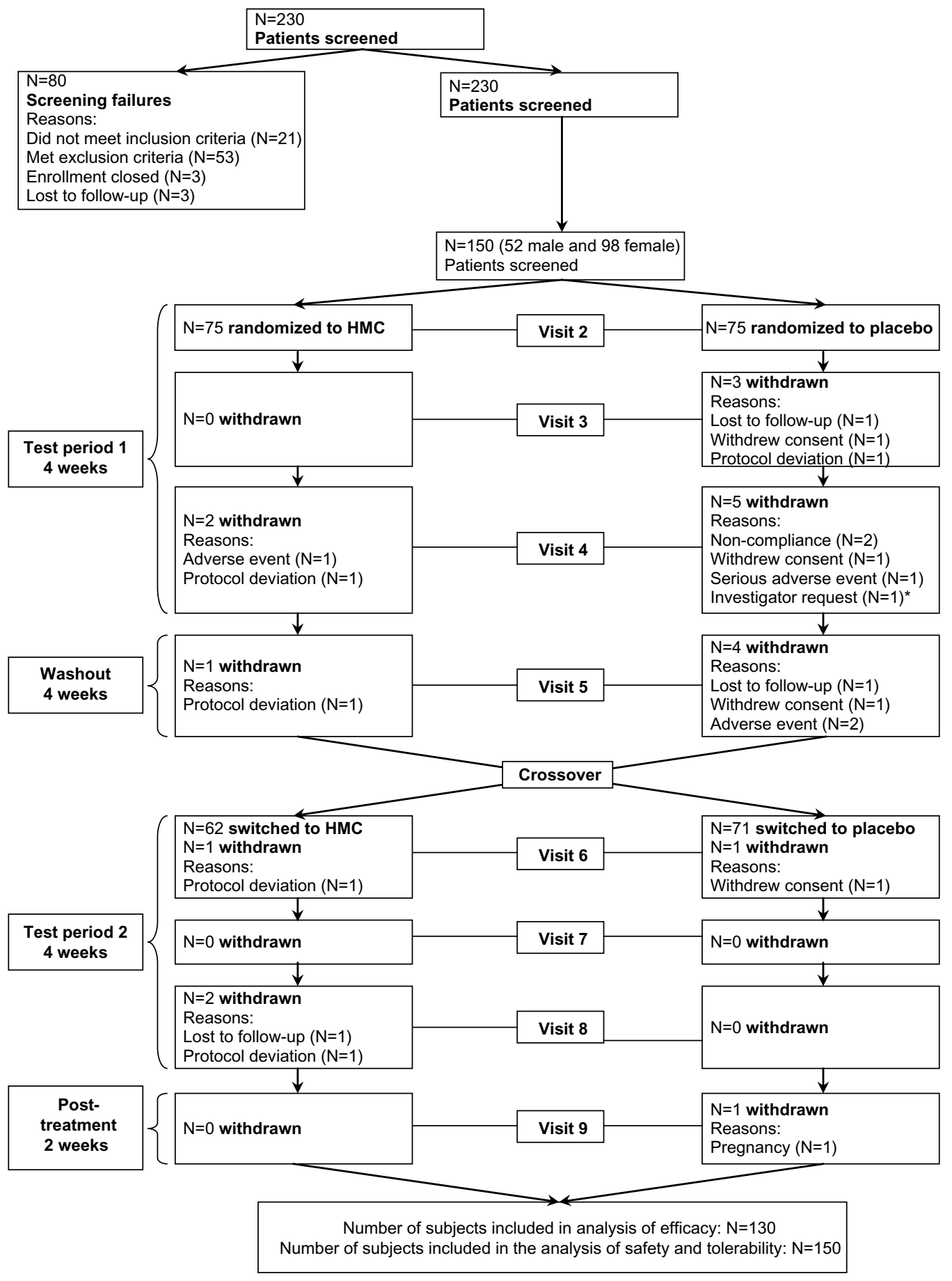

Figure I Flow diagram of the disposition of subjects screened and enrolled into the study.

Note: *Participant had elevated ferritin levels $(>500 \mathrm{mg} / \mathrm{L})$ at baseline and was withdrawn from the study at the request of the investigator.

Abbreviation: HMC, hydrothermal mineral complex.

A weight-dependent dose was not used for this study, to comply with regulatory requirements from NHPD. However, more than $70 \%$ of participants in both groups were overweight and obese, and were thus taking dosages of HMC that were below the manufacturer's recommendation. Participant demographics were similar between groups, and participants had treatment compliance $>95 \%$.

\section{WOMAC ${ }^{\mathrm{TM}}$ scores}

While there were no significant between-group differences in WOMAC ${ }^{\mathrm{TM}}$ pain, the primary endpoint, nor on physical function scores during the 4-week treatment period (Figure 2), analysis of secondary endpoints revealed differences, as did subgroup analysis by BMI categories. The HMC group showed an improvement in WOMAC ${ }^{\mathrm{TM}}$ stiffness from baseline to 
Table I Demographics and characteristics at screening of all subjects completing the study $(\mathrm{N}=\mathrm{I50})$

\begin{tabular}{|c|c|c|c|}
\hline & $\begin{array}{l}\text { Placebo } \rightarrow \text { HMC } \\
(\mathrm{N}=75)\end{array}$ & $\begin{array}{l}\text { HMC } \rightarrow \text { placebo } \\
(\mathrm{N}=75)\end{array}$ & $P$-value \\
\hline & {$[N]$ Mean \pm SD } & {$[N]$ Mean \pm SD } & \\
\hline & SEM & & \\
\hline & Median (Min, Max) & Median (Min, Max) & \\
\hline \multirow[t]{3}{*}{ Age, years } & [75] $45.28 \pm 12.97$ & [75] $44.7 \mathrm{I} \pm \mathrm{II} .52$ & $0.78^{\mathrm{a}}$ \\
\hline & 1.50 & 1.33 & \\
\hline & $46.00(19.00,65.00)$ & $48.00(18.00,64.00)$ & \\
\hline \multirow[t]{3}{*}{ Mean systolic blood pressure, $\mathrm{mmHg}$} & {$[75] \mid 115.52 \pm 13.15$} & {$[75] \mid I 4.56 \pm 13.39$} & $0.66^{\mathrm{a}}$ \\
\hline & 1.52 & 1.55 & \\
\hline & II $4.00(91.00,141.00)$ & $111.00(91.00,148.00)$ & \\
\hline \multirow[t]{3}{*}{ Mean diastolic blood pressure, $\mathrm{mmHg}$} & {$[75] 74.95 \pm 8.98$} & [75] $73.73 \pm 8.75$ & $0.40^{\mathrm{a}}$ \\
\hline & 1.04 & 1.01 & \\
\hline & $76.00(59.00,95.00)$ & $72.00(58.00,93.00)$ & \\
\hline \multirow[t]{3}{*}{ Mean heart rate, bpm } & {$[75] 68.83 \pm 6.96$} & {$[75] 68.12 \pm 6.25$} & $0.5 \mathrm{I}^{\mathrm{a}}$ \\
\hline & 0.80 & 0.72 & \\
\hline & $68.00(56.00,84.00)$ & $67.00(57.00,84.00)$ & \\
\hline \multirow[t]{3}{*}{ Height, cm } & [75] $167.76 \pm 8.85$ & [75] $167.36 \pm 9.03$ & $0.78^{\mathrm{a}}$ \\
\hline & 1.02 & 1.04 & \\
\hline & $167.00(150.50,185.30)$ & $166.50(148.00,187.30)$ & \\
\hline \multirow[t]{3}{*}{ Weight, kg } & [75] $84.86 \pm 22.03$ & [75] $83.39 \pm 21.52$ & $0.68^{\mathrm{a}}$ \\
\hline & 2.54 & 2.48 & \\
\hline & $82.10(50.80,158.50)$ & $82.40(45.80,145.70)$ & \\
\hline \multirow[t]{4}{*}{$\mathrm{BMI}, \mathrm{kg} / \mathrm{m}^{2}$} & {$[75] 30.01 \pm 6.83$} & [75] $29.59 \pm 6.52$ & $0.70^{\mathrm{a}}$ \\
\hline & 0.79 & 0.75 & \\
\hline & $28.50(19.50,54.80)$ & $28.60(17.40,50.40)$ & \\
\hline & $f / n(\%)$ & f/n (\%) & \\
\hline \multicolumn{4}{|l|}{ Age, years } \\
\hline$<30$ & I0/75 (13.33\%) & $10 / 75$ (13.33\%) & $0.94^{b}$ \\
\hline $30-49$ & $33 / 75$ (44.00\%) & $35 / 75$ (46.67\%) & \\
\hline$\geq 50$ & $32 / 75$ (42.67\%) & $30 / 75$ (40.00\%) & \\
\hline \multicolumn{4}{|l|}{$\mathrm{BMI}$ at screening, $\mathrm{kg} / \mathrm{m}^{2}$} \\
\hline$<25$ & I7/75 (22.67\%) & $19 / 75$ (25.33\%) & $0.78^{b}$ \\
\hline $25-29.9$ & $27 / 75$ (36.00\%) & $23 / 75(30.67 \%)$ & \\
\hline$\geq 30$ & $31 / 75$ (4I.33\%) & $33 / 75$ (44.00\%) & \\
\hline \multicolumn{4}{|l|}{ Sex } \\
\hline Female & $46 / 75$ (61.33\%) & $52 / 75(69.33 \%)$ & $0.30^{\mathrm{b}}$ \\
\hline Male & $29 / 75$ (38.67\%) & $23 / 75(30.67 \%)$ & \\
\hline \multicolumn{4}{|l|}{ Ethnicity } \\
\hline Not Hispanic or Latino & $72 / 75$ (96.00\%) & 67/75 (89.33\%) & $0.12^{\mathrm{b}}$ \\
\hline Hispanic or Latino & $3 / 75$ (4.00\%) & $8 / 75$ (10.67\%) & \\
\hline \multicolumn{4}{|l|}{ Alcohol use } \\
\hline None & I6/75 (21.33\%) & $22 / 75(29.33 \%)$ & $0.72^{\mathrm{b}}$ \\
\hline Occasional & $35 / 75$ (46.67\%) & $30 / 75(40.00 \%)$ & \\
\hline Weekly & $21 / 75(28.00 \%)$ & $20 / 75$ (26.67\%) & \\
\hline Daily & $3 / 75$ (4.00\%) & $3 / 75$ (4.00\%) & \\
\hline
\end{tabular}

Notes: ${ }^{a}$ Between-group statistical comparisons were conducted using a paired $t$-test; between-group statistical comparisons were conducted using a chi-square test. Probability values $P \leq 0.05$ are significant.

Abbreviations: BMI, body mass Index; f, frequency; HMC, hydrothermal mineral complex; Max, maximum; Min, minimum; SD, standard deviation; SEM, standard error of the mean.

weeks 2 and 4, while the placebo group showed an improvement from baseline to week 2 and then plateaued at week 4 (Figure 3). During the washout period, the HMC group had an increase in stiffness score, while those on placebo did not, leading to significant difference between groups at week $6(P=0.05)$.
There was no sequence effect in any domain except in the physical function scores at week $2(P=0.05)$ and week 6 $(P=0.04)$. There was a period effect in the stiffness domain at week $2(P<0.001)$, week $4(P<0.001)$, week 6 $(P<0.001)$, and in the physical function domain at week $6(P=0.03)$. 


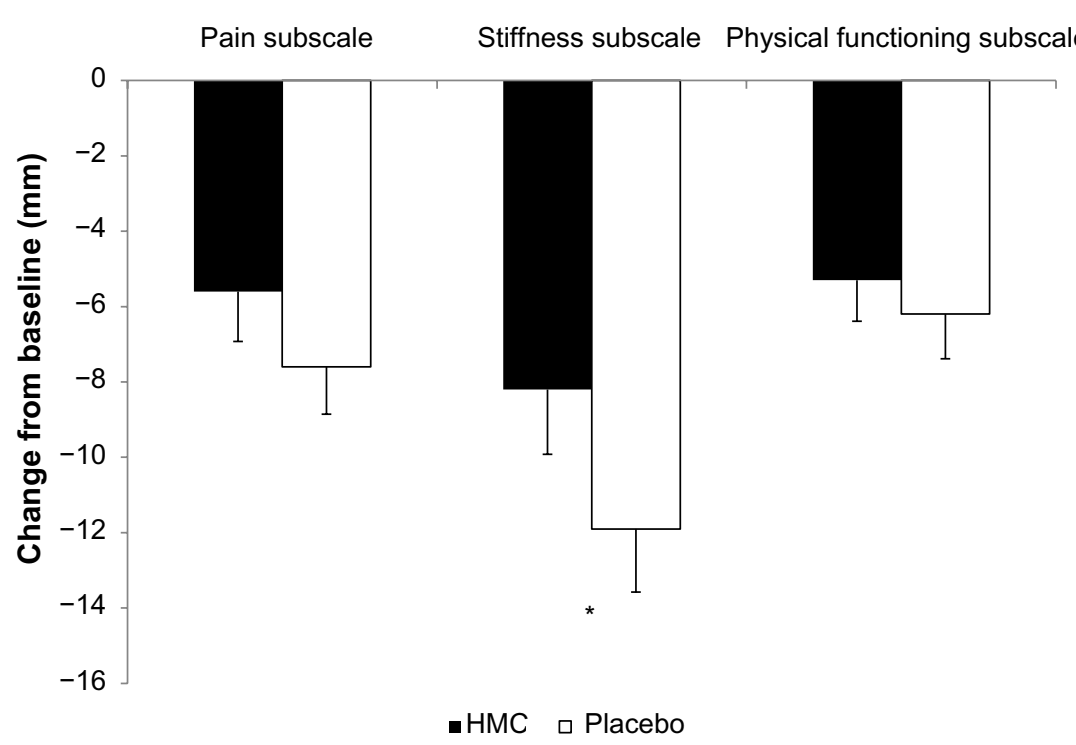

Figure 2 Effect of treatment on WOMAC TM subscales from baseline to week 4 for both test periods.

Notes: The change from baseline in WOMACTM scores between HMC (black) and placebo (white). *During the washout period, HMC group had an increase in stiffness score, while those on placebo did not, leading to significant difference between groups at week $6(P=0.05)$.

Abbreviation: HMC, hydrothermal mineral complex.

Within groups, both HMC and placebo group participants reported significant improvements in pain, physical function, and stiffness $(P<0.001)$.

\section{SF-36 scores}

After 4 weeks of supplementation, significant improvements in SF-36 physical function $(P=0.02)$ and total scores $(P=0.05)$ were reported by participants on HMC compared with placebo (Figure 4). Additionally, improving trends in total physical health $(P=0.06)$ and social functioning $(P=0.09)$ were reported by participants on HMC versus placebo (Figure 4).

There were no significant differences in the domains of role limitation due to physical health, limitations due to emotional problems, vitality/energy, mental health/emotional well-being, bodily pain, general health, and total mental health between $\mathrm{HMC}$ and placebo groups.

Within groups, participants on HMC reported significant improvements in SF-36 scores for physical function $(P<0.001)$, role limitation due to physical health $(P=0.01)$, vitality/energy $(P=0.003)$, social functioning $(P=0.01)$, bodily pain $(P<0.001)$, total SF-36 scores $(P<0.001)$, total physical health $(P<0.001)$, and total mental health $(P=0.02)$. Participants on placebo reported improvements only in bodily pain $(P<0.001)$, general health $(P=0.01)$, and total physical health $(P=0.04)$. Within groups, the improvements in scores from baseline to week 4 for all domains of the SF-36, with the exception of general health, were greater for participants on HMC compared with those on placebo.
On subgroup analysis by BMI, the greatest efficacy of HMC was in normal weight participants followed by those who were overweight. Normal weight participants on HMC showed a trend toward a decrease in the WOMAC ${ }^{\text {TM }}$ pain subscale from baseline to week $4(P=0.107)$, while there was a reported increase in pain for those on placebo (data not shown). There were no significant between-group differences in WOMAC ${ }^{\text {TM }}$ pain scores in either the overweight or obese participants in this study. This suggests perhaps that the dosage used in the study was adequate for normal weight participants but that dose escalation would be more appropriate for those with higher BMI. On subgroup analysis by age, participants less than 30 years of age performed better in the WOMACTM pain domain compared with those aged $30-49$, and those 50 years and older. However, the subgroup of participants less than 30 years of age also showed less prevalence of overweight and obesity. This observation further bolsters the escalation of dose according to BMI.

\section{Minimal clinically important improvement (MClI)}

The number of participants meeting MCII in WOMACTM pain scores increased from $28 \%$ to $41 \%$ from baseline to week 4 for subjects on $\mathrm{HMC}$, and decreased to $37 \%$ during the washout period (Figure 5A). Forty-four percent of participants on placebo responded by week 2 and continued to remain stable through week 4 as well as the washout period. On subgroup analysis by BMI, $35 \%$ of normal weight participants on $\mathrm{HMC}$ versus $8 \%$ of participants on placebo 

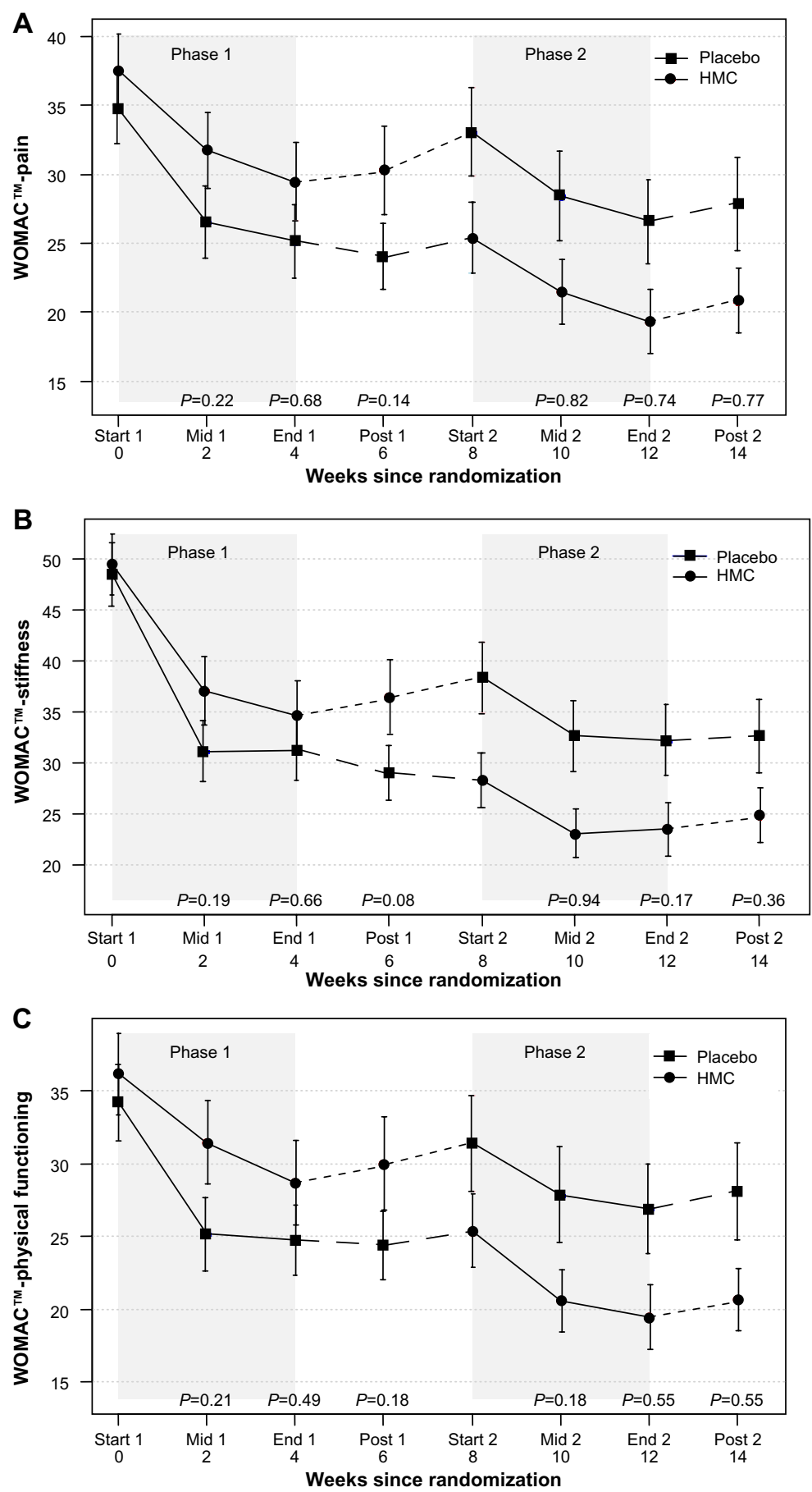

Figure 3 Effect of treatment on WOMACTM subscale scores by treatment phase.

Notes: The effect of placebo (squares) and HMC (circles) on WOMAC TM (A) pain, (B) stiffness, and (C) physical functioning subscale scores by treatment phase for all participants completing the study $(\mathrm{N}=130)$. WOMAC ${ }^{\mathrm{TM}}$ pain, stiffness, and physical functioning subscale scores did not return to baseline values following the washout period (week 8).

Abbreviation: HMC, hydrothermal mineral complex.

met $\mathrm{MCII}$ for pain $(P=0.104)$, demonstrating greater efficacy of $\mathrm{HMC}$ (Figure 5B).

Participants on HMC who were below 30 years of age and meeting the MCII for WOMACTM pain scores increased from $75 \%$ to $100 \%$ from baseline to week 4 , versus an increase from $20 \%$ to $40 \%$ for those on placebo
$(P=0.035)$. Participants below 30 years of age reported greater decreases for WOMACTM stiffness from baseline to week 4 versus those on placebo. Participants between 30 and 49 years of age showed decreases in WOMACтM stiffness from baseline to week 2, and from week 2 to week 4. 


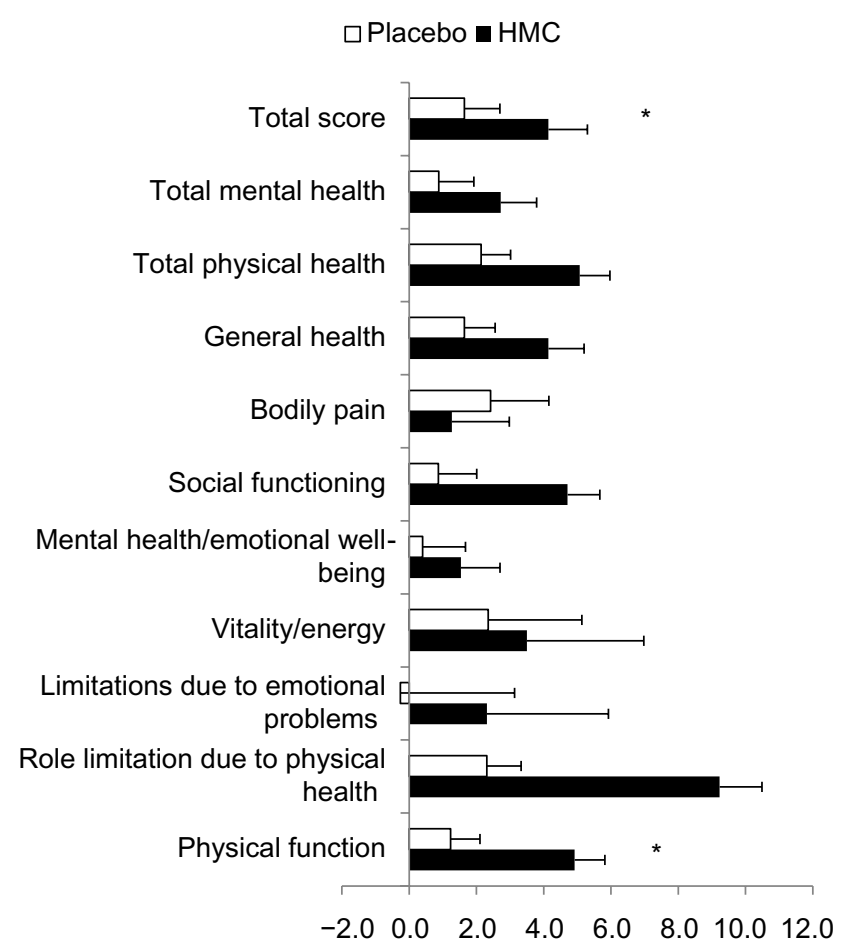

Change in score from baseline

Figure 4 Effect of treatment on SF-36 domain scores from baseline to week 4 for both test periods.

Notes: SF-36 domain scores between HMC (black) and placebo (white). *Significant improvement in SF-36 total $(P=0.05)$ and physical function $(P=0.02)$ scores were reported by participants in HMC group compared with placebo.

Abbreviations: HMC, hydrothermal mineral complex; SF-36, 36-Item Short Form Health Survey.

\section{Inflammation outcome measure}

There were no significant differences between treatment groups in either hsCRP, IL-6, or TNF- $\alpha$. TNF- $\alpha$ increased by $25 \%$ from baseline to week 4 in the placebo group but only by $15 \%$ when on HMC ( $P=0.07)$, suggesting a positive trend.

\section{Adherence, safety analysis, and adverse events}

Of the 150 randomized participants, 130 completed both periods of the crossover. Significantly more participants receiving placebo withdrew from the study than those on HMC $(P=0.03)$. On analysis of 3-day food diaries, the dietary habits of participants on HMC and placebo were similar with respect to number of daily servings of vegetables, fruits, grains, milk and milk products, and meat and meat alternatives consumed.

There were no differences between HMC and placebo groups in blood pressure, heart rate, weight, BMI, or any of the hematological or general blood chemistry parameters assessed, including electrolytes and kidney and liver
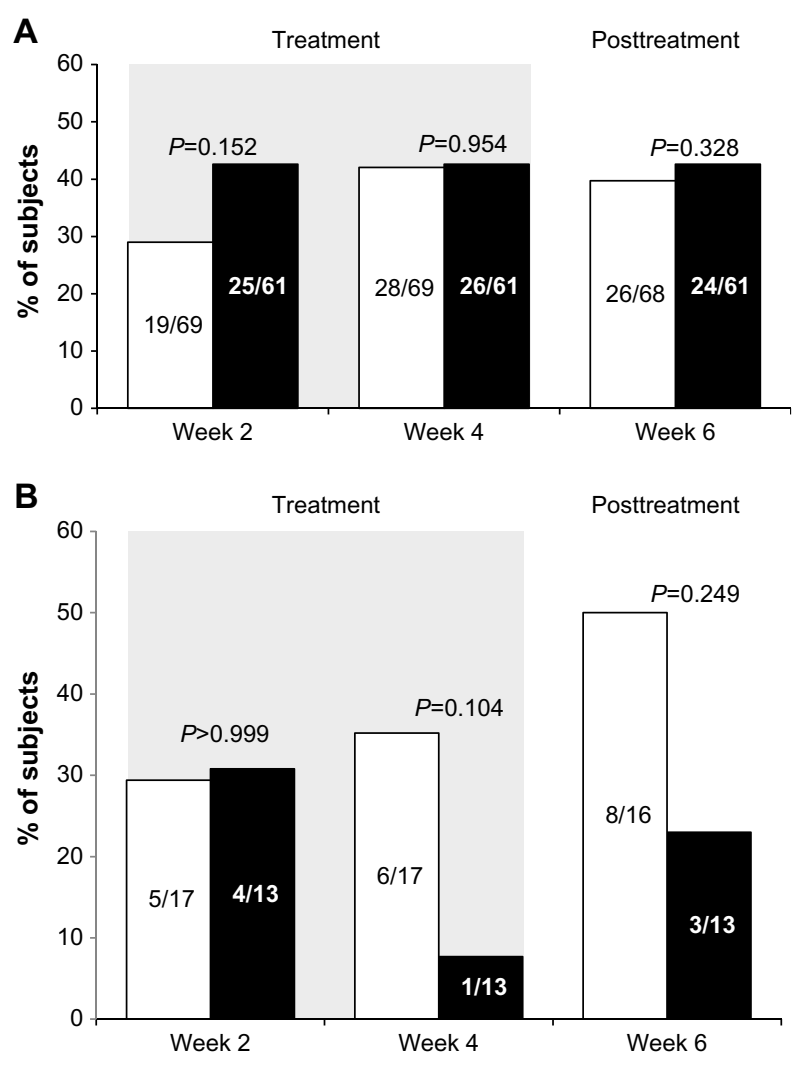

Figure 5 Effect of treatment on MCll.

Notes: Number of participants achieving the MCII in WOMAC ${ }^{\mathrm{TM}}$ pain (at least $40.8 \%$ improvement in pain scores from baseline) for (A) all subjects completing the study and (B) normal weight participants (BMI $<25 \mathrm{~kg} / \mathrm{m}^{2}$ ). Placebo group (black) and $\mathrm{HMC}$ group (white). Fractions represent the number of participants achieving the $\mathrm{MCll}$ in WOMACTM pain over the total number of participants.

Abbreviations: BMI, body mass index; HMC, hydrothermal mineral complex; MCll, minimal clinically important improvement.

function markers. Of the nine adverse events that were assessed as possibly related to the study product, eight were in the placebo group and one in the HMC group. The adverse event assessed as possibly related to HMC was bloating, which was also reported by one subject on placebo. These adverse events resolved prior to the end of the study. One adverse event was assessed as having a probable relationship to HMC (allergic reaction), but this was resolved prior to the end of the supplementation with HMC, suggesting that it was not causative of the treatment. There were no statistically significant differences in the number of participants reporting adverse events between the supplementation groups. Administration of up to three capsules of HMC per day ( $2 \mathrm{~g} /$ day) for up to 4 weeks was found to be safe in the population of participants investigated in this study.

\section{Discussion}

Well-designed, good-quality research studies on the efficacy of natural health products for OA are limited. 
The recommendations are for OA clinical trials to be designed as parallel studies that are single-joint, randomized, double-blind, and placebo-controlled, though crossover studies are also considered to be appropriate. ${ }^{21}$

The main benefit of a crossover study in which study participants serve as their own controls, is that confounding factors involved in comparing study and control groups are avoided. ${ }^{22}$ Furthermore, smaller sample sizes are sufficient to achieve the statistical power that is required to demonstrate treatment effect. However, the washout period in a crossover study must be sufficiently long to rule out any carryover effect in order to see efficacy. ${ }^{22}$ Another concern with crossover studies is that the period effect may extend over and above the treatment effect. ${ }^{22}$

The current study was conducted as a single (knee) joint, controlled, randomized, double-blind crossover study, but this design created complications for assessing trial results largely due to participants not returning to the original baseline values for WOMAC ${ }^{\text {TM }}$ domains after the washout (Figure 4). The placebo group was in better WOMACTM condition with regard to pain relief at the end of the first period and remained at that state during the washout period, while the HMC group reverted to higher scores (worse condition) during washout. This tilted the advantage toward placebo for the second period. Consequently, it was not surprising that the results from the first period of the crossover were not duplicated in the second period; therefore, a clear assessment of product performance could not be made when the results of the two periods were analyzed.

The combination of better placebo performance during the first period of the crossover, incomplete return to original scores during washout, with no return at all for the placebo group, and the possible presence of a floor effect probably gave the placebo an unfair advantage during the second period in this study. As there was no previous information on the required washout for $\mathrm{HMC}$, it is possible that the washout period of 4 weeks was too short for participants to return to baseline scores for their WOMAC ${ }^{\text {TM }}$ domains. In addition, significantly more participants dropped out of the study when they were on placebo compared with HMC. A noticeable deterioration in condition when the active treatment was withdrawn, but not for the placebo, as well as a significantly lower dropout rate when participants were on the active treatment, suggests that HMC may play a role in pain-relief, stiffness, and physical function.

The efficacy of HMC versus placebo could not be assessed adequately in this study with the crossover design. Similar problems with crossover clinical trials of OA have been reported in the literature, and in such cases, statistical significance could only be demonstrated by analyzing the treatment period before crossover. ${ }^{23}$ When the first period of the crossover was analyzed as a parallel study, there were no statistically significant differences in WOMAC тм pain, stiffness, or physical function subscale scores between participants on HMC and those on placebo at a uniform dose across all BMI categories during the 4-week treatment period (data not shown).

OA is considered one of the leading causes of disability and a frequent source of chronic pain, with individuals reporting the presence of depressive symptoms and an overall lower health-related quality of life. ${ }^{24,25}$ Mean scores for SF-36 limitations due to mental health/emotional wellbeing increased significantly for participants on HMC after 4 weeks of treatment compared with placebo, perhaps due to the improvements in the scores for SF-36 physical function, role limitation due to physical health, role limitation due to emotional health, vitality/energy, mental health/ emotional well-being, social functioning, bodily pain, and general health domains. These significant between-group improvements across various SF-36 domains were absent in participants receiving placebo, and indicated that supplementation improved the quality of life for those suffering from OA.

While the role of inflammation in OA pathogenesis is not clear, serum levels of TNF- $\alpha$ are associated with knee cartilage loss, and there is suggestion that low-level inflammation plays a role in the pathogenesis of knee OA. ${ }^{26}$ In addition, change in serum TNF- $\alpha$ has been found to be positively associated with change in knee pain, and systemic inflammation is reported to be an independent predictor of worsening knee pain over 5 years. ${ }^{27}$ Though there were no significant differences in levels between groups, participants on HMC had a lower increase in serum TNF- $\alpha$ levels compared to those on placebo, possibly suggesting reduced progression of inflammation. Enrollment criteria for this study, based on the ACR criteria, are in line with that reported in the literature and are frequently used in clinical studies on OA. ${ }^{28,29}$ Radiological score and joint effusions were not assessed prior to enrollment to assess OA pathogenesis, thus it is possible that participants with both early- and later-stage OA were enrolled in this study. Further research that includes radiography, for enrollment, in an adequately powered parallel study is warranted to verify these results.

This study did not exclude subjects based on BMI and study demographics after enrollment showed that the majority of participants were overweight or obese. However, it was clear from the results that the responses to $\mathrm{HMC}$ were strongest among normal weight participants. The 
weaker responses to HMC among overweight and obese participants may be due to inadequate dosage, as HMC is dosage-dependent, and dose escalation is required for subjects with higher BMI, consistent with the manufacturer's recommendation. Due to regulatory constraints, the dosage of HMC had to be restricted to a predetermined uniform dose, and all participants received the same dose regardless of BMI. A dosage study on the HMC has since been conducted, and the results indicated that HMC was safe at higher dosages (unpublished). Another possibility for the difference in response is that the pathogenesis of OA may be regulated by different mechanisms in overweight and obese participants, compared with normal weight participants. It has been long known that obesity is an independent risk for the development of symptomatic OA of the knee. ${ }^{30}$ Although the exact mechanism is still not fully understood, recent studies suggest that the contribution of obesity to OA is not just in excessive loads on weight-bearing joints, but also alterations in levels of hormones, cytokines, and growth factors from adipose tissue that may contribute to inflammation and cartilage degradation, and lead to joint degeneration. ${ }^{30,31}$

Stratification by age was not planned for this study. Given that primary OA is much more prevalent in the elderly, it is interesting to note that the study demographics showed the mean age of participants to be 45 years of age. Of the 150 participants enrolled, though $55 \%$ were $\geq 45$ years and $45 \%$ were below 45 years of age, $75 \%$ of the cohort had primary OA. Subgroup analysis by primary or secondary OA showed no significant differences in the efficacy of HMC between groups. Furthermore, as this was a crossover study, the whole cohort, regardless of OA type, received both placebo and the HMC.

There is a strong placebo effect in OA studies, regardless of sample size or the quality of the study design. In fact, Zhang et $\mathrm{al}^{32}$ reported that placebo is especially effective for patient-reported OA outcomes such as pain, stiffness, and function as well as for the physician's global assessment. The main determinants of the placebo effect are the expected strength of the treatment, baseline symptom severity, route of delivery, and the sample size. Furthermore, the baseline pain score is a predictor of the placebo effect, with a positive relationship existing between the baseline pain scores and the placebo effect. ${ }^{32}$ The WOMAC ${ }^{\text {TM }}$ results in the current study are consistent with the OA literature, where high placebo effects have been reported. ${ }^{23}$ The 1,500-subject, 12-week GAIT (Glucosamine/chondroitin Arthritis Intervention Trial) reported a placebo effect of $60 \%$ based on a $20 \%$ improvement in the MCII for the WOMAC ${ }^{\text {TM }}$ pain score. ${ }^{23}$
In this study, a placebo effect of $43 \%$ was seen based on a $40.8 \%$ improvement in the MCII for the WOMAC ${ }^{\text {TM }}$ pain score. ${ }^{20}$ These results are noteworthy considering a lower placebo effect was observed despite a smaller sample size and shorter intervention period. A run-in period may alleviate the placebo effect and improve the interpretation of the study results. Since OA is a long-term disease, increasing the study length for future studies may be warranted to achieve better separation and increased visibility between the efficacy of HMC compared with placebo, and also to evaluate the longterm efficacy of HMC.

\section{Conclusion}

The results of the study were mixed, in part due to limitations caused by the study design being changed from a weightdependent dosage to a uniform dosage, the higher BMI of the majority of recruited participants, and a washout period that may have been shorter than appropriate. Although this crossover study found no significant differences between the HMC and placebo supplementation groups in WOMACTM pain, stiffness, or physical function subscale scores, all three subscale scores deteriorated during the 2-week posttreatment period in the HMC group, which was not seen in the placebo group. Supplementation with HMC was found to have significant improvement in SF-36 physical function and total scores, as well as improving trends in total physical health, and social functioning scores, thus improving the overall quality of life. At the dosage of $2 \mathrm{~g}$ /day that was used in the study, HMC was most effective in normal weight participants. The results that were obtained may be, in part, due to the majority of the study population having BMI that was higher than recommended for adequate product dosage. An increased dose of HMC may be required to elicit efficacy in North American subjects with a higher BMI to have a positive effect on OA symptoms.

\section{Acknowledgments}

We wish to thank the volunteers who took part in this study for their willingness and diligence in complying with the study protocol. This study was conducted at KGK Synergize Inc. (London, ON, Canada) under the supervision of the Medical Director Dale Wilson, MD. The authors wish to thank Sonya Barss for overseeing the conduct of the study, Dr John Pezzullo for statistical advice and analysis, and Eh-Sanus Fahim for overseeing data management. We thank Joshua Baisley for regulatory and quality activities, and for his technical support. We wish to thank Hui Jun Chew for her diligence in reviewing and editing the manuscript. This study was sponsored by SierraSil Health Inc. (Vancouver, 
BC, Canada). SierraSil was involved in designing the protocol and had no involvement in conducting the study or collecting or analyzing the data.

\section{Disclosure}

ME and NG are full-time employees of KGK Synergize Inc. DW is the medical director and consultant for KGK Synergize Inc. ME, DW, and NG have no relationships with or financial interests in any commercial companies related to this study or article.

\section{References}

1. Lawrence RC, Felson DT, Helmick CG, et al. Estimates of the prevalence of arthritis and other rheumatic conditions in the United States. Part II. Arthritis Rheum. 2008;58(1):26-35.

2. Buckwalter JA, Saltzman C, Brown T. The impact of osteoarthritis: implications for research. Clin Orthop Relat Res. 2004;(Suppl 427): S6-S15.

3. Sengupta K, Alluri KV, Satish AR, et al. A double blind, randomized, placebo controlled study of the efficacy and safety of 5-Loxin for treatment of osteoarthritis of the knee. Arthritis Res Ther. 2008;10(4):R85.

4. Nicholson S, Dickman K, Maradiegue A. Reducing premature osteoarthritis in the adolescent through appropriate screening. J Pediatr Nurs. 2009;24(1):69-74.

5. Leung GJ, Rainsford KD, Kean WF. Osteoarthritis of the hand I: aetiology and pathogenesis, risk factors, investigation and diagnosis. J Pharm Pharmacol. 2014;66(3):339-346.

6. Hochberg MC, Altman RD, Brandt KD, et al. Guidelines for the medical management of osteoarthritis. Part II. Osteoarthritis of the knee. American College of Rheumatology. Arthritis Rheum. 1995;38(11):1541-1546.

7. Felson DT, Lawrence RC, Dieppe PA, et al. Osteoarthritis: new insights. Part 1: the disease and its risk factors. Ann Intern Med. 2000;133:635-646.

8. Recommendations for the medical management of osteoarthritis of the hip and knee: 2000 update. American College of Rheumatology Subcommittee on Osteoarthritis Guidelines. Arthritis Rheum. 2000;43(9):1905-1915.

9. Pendleton A, Arden N, Dougados M, et al. EULAR recommendations for the management of knee osteoarthritis: report of a task force of the Standing Committee for International Clinical Studies Including Therapeutic Trials (ESCISIT). Ann Rheum Dis. 2000;59(12): 936-944.

10. Singh G. Recent considerations in nonsteroidal anti-inflammatory drug gastropathy. Am J Med. 1998;105(1B):31S-38S.

11. Griffin MR. Epidemiology of nonsteroidal anti-inflammatory drugassociated gastrointestinal injury. Am J Med. 1998;104:23S-29S.

12. Wright JM. Double-edged sword of COX-2 selective NSAIDs. CMAJ. 2002;167:1131-1137.

13. Haq I, Murphy E, Dacre J. Osteoarthritis. Postgrad Med J. 2003;79(933): 377-383.

14. Little CV, Parsons T, Logan S. Herbal therapy for treating osteoarthritis. Cochrane Database Syst Rev. 2001;1:CD002947.

15. Cronan TA, Kaplan RM, Posner L, Blumberg E. Prevalence of the use of unconventional remedies for arthritis in a metropolitan community. Arthritis Rheum. 1989;32(12):1604-1607.
16. Boisset M, Fitzcharles M. Alternative medicine use by rheumatology patients in a universal health care setting. $J$ Rheumatol. 1994;21(1):148-152.

17. Miller MJS, Ahmed S, Bobrowski P, Haqqi TM. Suppression of Human cartilage degradation and chondrocyte activation by a unique mineral supplement (SierraSili ${ }^{\mathrm{TM}}$ ) and a cat's claw extract, Vincaria ${ }^{\circledR}$. J Amer Nutr Assoc. 2004;7:32-39.

18. Miller MJ, Mehta K, Kunte S, et al. Early relief of osteoarthritis symptoms with a natural mineral supplement and a herbomineral combination: a randomized controlled trial [ISRCTN38432711]. J Inflamm (Lond). 2005;2:11.

19. Schulz KF, Altman DG, Moher D. CONSORT 2010 Statement: updated guidelines for reporting parallel group randomised trials. J Clin Epidemiol. 2010;63(8):834-840.

20. Tubach F, Ravaud P, Baron G, et al. Evaluation of clinically relevant changes in patient reported outcomes in knee and hip osteoarthritis: the minimal clinically important improvement. Ann Rheum Dis. 2005;64(1):29-33.

21. Altman R, Brandt K, Hochberg M, et al. Design and conduct of clinical trials in patients with osteoarthritis: recommendations from a task force of the Osteoarthritis Research Society. Results from a workshop. Osteoarthritis Cartilage. 1996;4(4):217-243.

22. Wellek S, Blettner M. On the proper use of the crossover design in clinical trials: part 18 of a series on evaluation of scientific publications. Dtsch Arztebl Int. 2012;109(15):276-281.

23. Clegg DO, Reda DJ, Harris CL, et al. Glucosamine, chondroitin sulfate, and the two in combination for painful knee osteoarthritis. $N$ Engl $J$ Med. 2006;354(8):795-808.

24. Lin EH. Depression and osteoarthritis. Am J Med. 2008;121(11 Suppl 2): S16-S19.

25. Felson DT, Zhang Y. An update on the epidemiology of knee and hip osteoarthritis with a view to prevention. Arthritis Rheum. 1998;41(8):1343-1355.

26. Stannus O, Jones G, Cicuttini F, et al. Circulating levels of IL-6 and TNF-alpha are associated with knee radiographic osteoarthritis and knee cartilage loss in older adults. Osteoarthritis Cartilage. 2010;18(11):1441-1447.

27. Stannus OP, Jones G, Blizzard L, Cicuttini FM, Ding C. Associations between serum levels of inflammatory markers and change in knee pain over 5 years in older adults: a prospective cohort study. Ann Rheum Dis. 2013;72(4):535-540.

28. Ruff KJ, Winkler A, Jackson RW, DeVore DP, Ritz BW. Eggshell membrane in the treatment of pain and stiffness from osteoarthritis of the knee: a randomized, multicenter, double-blind, placebo-controlled clinical study. Clin Rheumatol. 2009;28(8):907-914.

29. Kraemer WJ, Ratamess NA, Maresh CM, et al. Effects of treatment with a cetylated fatty acid topical cream on static postural stability and plantar pressure distribution in patients with knee osteoarthritis. J Strength Cond Res. 2005;19(1):115-121.

30. Sridhar MS, Jarrett CD, Xerogeanes JW, Labib SA. Obesity and symptomatic osteoarthritis of the knee. J Bone Joint Surg Br. 2012;94(4):433-440.

31. Vuolteenaho K, Koskinen A, Moilanen E. Leptin - a link between obesity and osteoarthritis. Applications for prevention and treatment. Basic Clin Pharmacol Toxicol. 2014;114(1):103-108.

32. Zhang W, Robertson J, Jones AC, Dieppe PA, Doherty M. The placebo effect and its determinants in osteoarthritis: meta-analysis of randomised controlled trials. Ann Rheum Dis. 2008;67(12):1716-1723. 


\section{Publish your work in this journal}

Open Access Rheumatology Research and Reviews is an international, peer-reviewed, open access journal, publishing all aspects of clinical and experimental rheumatology in the clinic and laboratory including the following topics: Pathology, pathophysiology of rheumatologica diseases; Investigation, treatment and management of rheumatological

Submit your manuscript here: http://www.dovepress.com/open-access-rheumatology-research-and-reviews-journal diseases; Clinical trials and novel pharmacological approaches for the treatment of rheumatological disorders. The manuscript management system is completely online and includes a very quick and fair peerreview system, which is all easy to use. Visit http://www.dovepress.com/ testimonials.php to read real quotes from published authors. 